\title{
Screening of Plants Hydro-Alcoholic Extracts from Kerman for their Inhibition of $\beta$-Glucuronidase Activity
}

\author{
Ahmad Gholamhoseinian ${ }^{1,2}$ (D), Fariba Sharififar ${ }^{3}$ (D) , Hamide Jalaeeian ${ }^{1,4}$, Beydolah Shahouzehi ${ }^{4,5}$,* (D) \\ 1 Department of Clinical Biochemistry, Kerman University of Medical Sciences, Kerman, Iran \\ 2 Physiology Research Center, Institute of Basic and Clinical Physiology Sciences, Kerman University of Medical Sciences, \\ Kerman, Iran \\ 3 Department of Pharmacognosy, School of Pharmacy, Kerman University of Medical Science, Kerman, Iran \\ 4 Student Research Committee, Kerman University of Medical Science, Kerman, Iran \\ 5 Cardiovascular Research Center, Institute of Basic and Clinical Physiology Sciences, Kerman University of Medical \\ Sciences, Kerman, Iran \\ * Correspondence: b.shahozehi@kmu.ac.ir; bshahouzehi@gmail.com;
}

Scopus Author ID 36470992900

Received: 15.06.2020; Revised: 4.07.2020; Accepted: 5.07.2020; Published: 9.07.2020

Abstract: In neonatal jaundice, $\beta$-glucuronidase converts conjugated bilirubin into the unconjugated form and increases its level in the blood. Many natural compounds have been identified as $\beta$ glucuronidase inhibitors. The aim of this study was to evaluate the effect of hydro-methanolic extracts of 100 plants on $\beta$-glucuronidase. The $\beta$-glucuronidase activity was measured by a spectrophotometric method using Phenolphthalein glucuronide and 4-nitrophenyl $\beta$-D-glucuronide. Kinetic study of the enzyme was performed in the presence and absence of the plant extract. It was revealed that from hydromethanolic (70\%) extracts, Rosa damascena and Ipomoea tricolor showed more than $85 \%$ inhibitory effect on $\beta$-glucuronidase. Rosa damascena showed competitive inhibition, and Ipomoea tricolor showed non-competitive inhibition. The $\mathrm{K}_{\mathrm{m}}$ and $\mathrm{V}_{\max }$ values for $\beta$-glucuronidase were $23.32 \mathrm{mM}$ and $0.814 \mathrm{mM} \mathrm{min}^{-1}$, respectively. When using 4-nitrophenyl $\beta$-D-glucuronide, Stevia and Cerasus avium showed more than $65 \%$ inhibitory effect on $\beta$-glucuronidase. Both Stevia and Cerasus avium showed non-competitive inhibition. The $\mathrm{K}_{\mathrm{m}}$ and $\mathrm{V}_{\max }$ values for $\beta$-glucuronidase were $16.98 \mathrm{mM}$ and $0.936 \mathrm{mM}$ $\min ^{-1}$, respectively. None of the plant extracts showed an activation effect on the enzyme. The data suggest that these plants might be good candidates for the treatment of neonatal jaundice and its related diseases.

Keywords: $\beta$-glucuronidase; Neonatal jaundice; Phenolphthalein Glucuronide; 4-Nitrophenyl $\beta$-Dglucuronide.

(C) 2020 by the authors. This article is an open-access article distributed under the terms and conditions of the Creative Commons Attribution (CC BY) license (https://creativecommons.org/licenses/by/4.0/).

\section{Introduction}

Jaundice is caused by high levels of bilirubin in the blood due to infection, genetic factors, and preterm delivery [1-4]. The jaundice is derived from the Greek word jaune, which is recognized by yellowing the skin and eye scrubs due to the increased amount of serum bilirubin pigments [3,5]. Jaundice can be detected with a wide variety of benign or threatening disorders. Bilirubin level above $2.5-5 \mathrm{mg} / \mathrm{dL}$ is a symptom of jaundice [3,6,7]. However, the central nervous system is at high risk when the unconjugated bilirubin level is between 10-20 $\mathrm{mg} / \mathrm{dL}$, which requires treatment $[2,4,7,8]$. Jaundice may be due to various diseases, including genetic syndrome, liver diseases, autoimmune disorders, gallstones, hepatitis, pancreatic 
cancer, papillary carcinoma, and medicines [3,9]. Severe jaundice can cause deafness, cerebral palsy, kernicterus, and other types of brain damage [10].

In neonatal jaundice, the level of unconjugated bilirubin increases. In other words, $\beta$ glucuronidase (EC 3.2.1.31), with its hydrolase activity, converts the conjugated bilirubin to unconjugated, which plays an important role in the development of neonatal jaundice $[3,4,11]$. The enzyme is produced by the intestinal $E$. coli, which has activity in both intestinal tissues and the bacteria. $\beta$-glucuronidase is found in animals, plants, and bacteria and causes $\beta$ glucuronide hydrolysis [12,13]. Intestines of neonates are free of bacteria and break down the conjugated bilirubin in the intestinal tract in adults and make it easier to remove it $[12,13]$. On the other hand, the activity of $\beta$-glucuronidase is high in the intestinal mucosa of the infants [7]. This results in the conversion of conjugated bilirubin into the unconjugated bilirubin in the intestines, which is reabsorbed through the intestinal epithelial cells and enters the intestinalhepatic cycle. This action increases the level of unconjugated bilirubin and causes jaundice in newborn infants $[4,7,14]$. On the other hand, $\beta$-glucuronidase increases CPT-11 activity, an anti-cancer drug, and plays an important role in various types of cancers, including colorectal, lung, breast, gastric, pancreatitis, cervix and ovarian cancers [15,16]. CPT-11 is converted to SN-38 in the liver by the carboxylesterase, and SN-38 is converted to SN-38G by the UTPglucuronyltransferase [16]. SN-38G passes through the digestive system and is considered as a substrate for the intestinal $\beta$-glucuronidase [16]. By converting SN-38G to SN-38, which is a topoisomerase, $\beta$-glucuronidase increases drug activity through fixing single-strand DNA breaks and cancer cell apoptosis [17].

In some cases, the enzyme activity has been reported to cause diarrhea [16]. Therefore, regulating the enzyme activity by increasing or decreasing activity can be effective in balancing the effectiveness of the drug. The use of plants as medicines has a long history [18-22]. Many studies on the inhibition of $\beta$-glucuronidase have been done in Korea [23], Japan [24], and Afghanistan [25].

In this study, we aimed to find hydro-methanolic extracts of 100 different plants to inhibit $\beta$-glucuronidase. Beta-glucuronidase inhibitors are well known for controlling various diseases, including hepatitis, hepatic necrosis, arthritis, and even colorectal cancer [8]. Dglucuno-1-4-lactone is currently one of the most important $\beta$-glucuronidase inhibitor, which is used in the treatment of several cancers, including ovarian, uterus, and prostate. The use of chemical drugs has always had some complications. $\beta$-glucuronidase also has two different properties: in some cases, its inhibition results in the treatment of the disease, while in some other cases increase in its activity is in benefit of the patient. Depending on the type of disease, patients should be advised to use or avoid using active substances. Considering the long history of plants used as medicines as well as biodiversity in different areas, we aimed to investigate the effect of hydro-methanolic extract of 100 plants on $\beta$-glucuronidase in order to find cheaper and safer new medicines in treatment and control of various diseases.

\section{Materials and Methods}

\subsection{Plants.}

One hundred plants were collected during summer 2016 and or purchased from the medicinal herbal markets in Kerman, and all of them were botanically identified by a botanist. A voucher specimen was deposited at the herbarium of the Herbal Medicines Research Center, Faculty of Pharmacy, Kerman University of Medical Sciences, Iran (Table 1). Those herbs 
which were purchased from Kerman medicinal markets; therefore, the codes are not known to the researchers.

Each of the plants was air-dried and grounded into a fine powder. Twenty grams of each plant powder was suspended in $200 \mathrm{~mL}$ of methanol (70\%), evenly soaked and stirred for one hour using the shaker, then placed at room temperature in dark conditions for 24 hours. The suspension was filtered and air-dried at room temperature. The resulting powders were stored in dark vials at $-20^{\circ} \mathrm{C}$ until use $[20,21,26]$.

\subsection{Enzyme assay.}

The $\beta$-glucuronidase, Phenolphthalein glucuronide, and 4-nitrophenyl $\beta$-D-glucuronide (4-NPG) were purchased from Sigma CO., USA. The $\beta$-glucuronidase activity was measured for each substrate by the spectrophotometric method [27]. In the method in which Phenolphthalein glucuronide was used as the substrate, 1 and $5 \mathrm{mg} / \mathrm{ml}$ of the extract was dissolved in methanol (10\%) and $0.1 \mathrm{ml}$ of the solution was added to $0.6 \mathrm{ml}$ of sodium acetate $1 \mathrm{M}$ and $0.2 \mathrm{ml}$ of phenolphthalein glucuronide $0.01 \mathrm{M}$. Mixture was incubated in $37{ }^{\circ} \mathrm{C}$ in a water bath for $5 \mathrm{~min}$. Negative control was prepared without the extract in a similar manner. Similarly, a blank sample was also prepared without the extract. $\beta$-glucuronidase $(0.1 \mathrm{ml})$ at a concentration of $2 \mathrm{mg} / \mathrm{ml}$ was added to the test tubes, and $0.1 \mathrm{ml}$ of water was added to the blank then incubated for $30 \mathrm{~min}$. To terminate the reaction, after 30 minutes, $1 \mathrm{ml}$ of the inhibitors (a mixture of $0.2 \mathrm{M}$ Glycine and $0.2 \mathrm{M}$ sodium chloride) were added to the tubes. Laspartic acid was used as a positive control [28].

In another method, in which 4-NPG was used as the second substrate, a solution of 10 $\mathrm{mg} / \mathrm{ml}$ of 4-NPG was prepared in phosphate buffer $(0.05 \mathrm{M}), \mathrm{pH} 7.20$. Sixty $\mu$ l of the substrate and $20 \mu \mathrm{l}$ of the extract was added to $50 \mu \mathrm{l}$ of the enzymatic solution $(2 \mathrm{mg} / \mathrm{ml}$ in phosphate buffer). The mixture was incubated in a $37^{\circ} \mathrm{C}$ water bath for $20 \mathrm{~min}$. Then $200 \mu \mathrm{l}$ of $\mathrm{Na}_{2} \mathrm{Co}_{3}$ $0.2 \mathrm{M}$ was added as a reaction inhibitor. Finally, the absorbance of the tubes was measured against distilled water at $405 \mathrm{~nm}$. Each test was performed 3 times, and the mean value was used as the inhibitory activity of the plant extract. L-aspartic acid was used as a positive control [27]. The inhibitory activity was calculated using the following formula [27,29]:

Inhibition $(\%)=[$ A Control-A Extract / A Control $] \times 100$

\subsection{Kinetic study.}

In order to measure the inhibition mode by hydro-methanolic extract with high activity, $\beta$-glucuronidase activity was assayed with: a) increasing concentrations of Phenolphthalein glucuronide the first substrate $(0.39,0.775,1.5,3.1$ and $6.2 \mathrm{mM})$ in the absence or presence of one of the extracts at two different extract concentrations (2.5 and $\left.5 \mathrm{mg} \cdot \mathrm{mL}^{-1}\right)$; b) increasing concentrations of 4-NPG as the second substrate $(0.5,0.793,1,1.578,2$ and $3.03 \mathrm{mM})$ in the absence or presence of one of the extracts at two different extract concentrations $(2.5$ and 5 $\left.\mathrm{mg} \cdot \mathrm{mL}^{-1}\right)$. Optimal doses of the extracts were determined based on the results from the inhibitory activity assay, as described above. The inhibition type for these extracts was determined by Lineweaver-Burk plot analysis [30].

\section{Results and Discussion}

Using Phenolphthalein glucuronide, Rosa damascena, and Ipomoea tricolor and showed more than $85 \%$ inhibitory effect on $\beta$-glucuronidase, while other plants extracts 
showed less than $85 \%$ inhibitory effect or even no inhibitory effect on this enzyme (Table 1). Using 4-nitrophenyl $\beta$-D-glucuronide, Stevia and Cerasus avium showed more than 65\% inhibitory effect on $\beta$-glucuronidase, while other plants extracts showed less than $65 \%$ inhibitory effect or even no inhibitory effect on this enzyme (Table 2).

Inhibition mode of two plants (Rosa damascena and Ipomoea tricolor) against Phenolphthalein glucuronide was analyzed by Lineweaver-Burk plots. The enzyme kinetics demonstrated a competitive inhibition by Rosa damascena. Enhancing the concentration of this extract showed an increase in the $\mathrm{K}_{\mathrm{m}}$, and no effect on $\mathrm{V}_{\max }$ values (Figure 1). While competitive inhibition on $\beta$-glucuronidase activity was observed by Ipomoea tricolor, enhancing the concentration of this extract showed a decrease in $\mathrm{V}_{\max }$ and $\mathrm{K}_{\mathrm{m}}$ values (Figure. 2). The $\mathrm{K}_{\mathrm{i}}$ values for Rosa damascena and Ipomoea tricolor were $0.276 \mathrm{mg} / \mathrm{ml}$ and 0.486 $\mathrm{mg} / \mathrm{ml}$, respectively. The inhibition mode of two plants (Stevia and Cerasus avium) against 4NPG was analyzed by Lineweaver-Burk plots. The enzyme kinetics demonstrated a noncompetitive inhibition by Stevia and Cerasus avium. Enhancing the concentration of these extracts showed a decrease in the $V_{\max }$ and no effect on $K_{m}$ values (Figures 3 and 4). The $K_{i}$ values for Stevia and Cerasus avium were $1.67 \mathrm{mg} / \mathrm{ml}$ and $1.8 \mathrm{mg} / \mathrm{ml}$, respectively.

In neonatal jaundice, the amount of non-conjugated bilirubin increases. $\beta$ glucuronidase converts conjugated bilirubin to non-conjugated and causing neonatal jaundice $[3,4,11]$. Severe jaundice can cause deafness, cerebral palsy, kernicterus, and other forms of brain damage [10]. $\beta$-glucuronidase also increases the activity of CPT-11, an anti-cancer drug, by converting SN-38G to SN-38 [15]. D-glucono-1-4-lactone is currently one of the most important $\beta$-glucuronidase inhibitor, which is used in the treatment of several cancers, including ovarian, uterus, and prostate [8]. However, the use of chemical drugs always has some complications. In this study, the inhibitory effect of hydro-ethanolic extracts of 100 plants on $\beta$-glucuronidase activity. Using Phenolphthalein glucuronide, the inhibitory effect of Rosa damascena and Ipomoea tricolor on $\beta$-glucuronidase were $94 \%$ and $86 \%$, respectively. Rosa damascena showed a competitive inhibition on the $\beta$-glucuronidase, but Ipomoea tricolor showed a non-competitive inhibition. Using 4-nitrophenyl $\beta$-D-glucuronide, the inhibitory effect of Stevia and Cerasus avium on $\beta$-glucuronidase was $75 \%$ and $68 \%$, respectively. Both plants demonstrated a non-competitive inhibition. Hydro-methanolic extract of Rosa damascena, Ipomoea tricolor, stevia, and Cerasus avium showed an inhibitory effect on $\beta$ glucuronidase. In this study, no plant showed an activating effect on $\beta$-glucuronidase. It is worth mentioning that several plants with less than $10 \%$ activating effect were found in this study, which is not enough to activate the $\beta$-glucuronidase.

$\beta$-glucuronidase released from bacteria, related to a higher incidence of Crohn's disease and colon cancer and, therefore, the inhibition of this enzyme released from bacteria living in the gut, is related to improving the health system [12]. Karak et al. (2019 and 2017) reported that plant extract from Swertia species and also Piper betle have $\beta$-glucuronidase inhibitory effects, which confirmed our findings $[18,19]$. In a study in which $\beta$-glucuronidase inhibitory effects of some plants were evaluated, they found that Mentha piperita strongly inhibited the enzyme activity (88.9\%), in our study we found that the inhibition is dependent on the substrate and Mentha piperita inhibited $\beta$-glucuronidase activity $63 \%$ and $9 \%$ when we used phenolphthalein glucuronide and 4-NPG as substrate, respectively. They used phenolphthalein glucuronide as the substrate and found higher inhibition activity by Mentha piperita that can be related to the type of extract used by them. In their study, they used the total flavonoid 
contents of Mentha piperita, but in the current study, we used a crude hydro methanolic extract of Mentha piperita [13].

The $\beta$-glucuronidase expression was up-regulated in patients with some cancers, including breast, lung, leukemia, and colon. Also, it up-regulated in diseases such as diabetes, HIV, and rheumatoid arthritis. It seems that $\beta$-glucuronidase can be considered as biomarkers in those diseases and also may be involved in their pathogenesis. Therefore, $\beta$-glucuronidase inhibition reduces those pathological conditions and attenuates their adverse consequences [8].

Table 1. Plants and their inhibitory effect on phenolphthalein glucuronide.

\begin{tabular}{|c|c|c|c|}
\hline Plants name & Family & Used part & Inhibition (\%) \\
\hline Rosa damascena & Rosaceae & Galls & 94 \\
\hline Ipomoea tricolor & Convolvulaceae & Galls & 86 \\
\hline Ziziphora tenuir & Lamiaceae & Leaves & 84 \\
\hline Nigella sativa $L$. & Ranunculaceae & Seeds & 83 \\
\hline Origanum majorana & Lamiaceae & Whole plant & 81 \\
\hline Syzygium aromaticus & Caryophyllaceae & Aerial part & 78 \\
\hline Huscoriaria & Anacardiaceae & Seeds & 77 \\
\hline Scrophularia striata & Compositae & Aerial part & 77 \\
\hline Olea europaea & Oleaceae & Leaves & 77 \\
\hline Zataria multiflora & Lamiaceae & Aerial part & 76 \\
\hline Lavandula angustifolia & Lamiaceae & Whole plant & 75 \\
\hline Rosa & Rosaceae & Galls & 75 \\
\hline Avena sativa & Grasses & Seeds and shell & 74 \\
\hline Echium amoenum & Boraginaceae & Galls & 74 \\
\hline Cerasus avium & Rosaceae & Fruit Tails & 73 \\
\hline Glycyrrhiza glabra & Legumes & Aerial part & 70 \\
\hline Myrtus communis & Myrtaceae & Leaves & 70 \\
\hline Equisetum arvense & Equisetaceae & Whole plant & 70 \\
\hline Tanacetum parthenium & Asteraceae & Galls & 69 \\
\hline Centaurea depressa & Grasses & Galls & 68 \\
\hline Papaver somniferum & Papaveraceae & Seeds & 67 \\
\hline Cucumis sativus & Cucurbitaceae & Seeds & 67 \\
\hline Otostegia persica & Violaceae & Aerial part & 65 \\
\hline Sanguisorba minor & Geraniacea & Aerial part & 64 \\
\hline Mentha piperita & Lamiaceae & Leaves & 63 \\
\hline Arctium lappa & Asteraceae & Whole plant & 62 \\
\hline Cubeb berries & Piperaceae & Fruits & 61 \\
\hline Phoenix dactylifera & Arecaceae & Galls andFloret & 61 \\
\hline Medicago sativa $L$. & Legumes & Seeds & 61 \\
\hline Matricaria recutita & Composite & Galls & 61 \\
\hline Hypericum perforayum & Labiatae & Aerial part & 60 \\
\hline Astragalus adsccendens & Psyllidae & Gum & 59 \\
\hline Crataegus oxyacanta & Rosaceae & Galls and Fruits & 58 \\
\hline Coriandrum sativum & Apiaceae & Aerial part & 58 \\
\hline Alhagi maurorum & Fabaceae & Gum & 57 \\
\hline Citrus aurantium & Rutaceae & Galls & 56 \\
\hline Crataegus oxyacanta & Astraceae & Whole plant & 55 \\
\hline Cotoneaster & Rosaceae & Gum & 53 \\
\hline Hyssopus officinalis & Lamiaceae & Plant flowers & 52 \\
\hline Calendula officinalis $L$. & Asteraceae & Whole plant & 51 \\
\hline Urticadioica & Urticaceae & Aerial part & 49 \\
\hline Punica granatum & Punicaceae & Skin & 49 \\
\hline Sesamum indicum & Pedaliaceae & Seeds & 48 \\
\hline Cinnamomum Zeylanicum & Lauraceae & Roots & 48 \\
\hline Stevia & Asteraceae & Leaves & 47 \\
\hline Rosmarinus officinalilis & Lamiaceae & Aerial part & 47 \\
\hline Artemisia absinthium & Compositae & Leafy branches & 44 \\
\hline Lippia citriodora & Verbenaceae & Leaves & 44 \\
\hline Allium sativum $L$. & Lily & Gland & 44 \\
\hline Polygonatum orientale Desf. & Umbelliferae & Roots & 41 \\
\hline Camellia sinensis & Theaceae & Leaves & 40 \\
\hline Mentha longifolia & Labiatae & Aerial part & 39 \\
\hline Achillea wilhelmsii & Compositae & Aerial part & 37 \\
\hline Lactuca sativa $L$. & Compositae & Seeds & 36 \\
\hline
\end{tabular}


https://doi.org/10.33263/BRIAC111.79217931

\begin{tabular}{|c|c|c|c|}
\hline Plants name & Family & Used part & Inhibition $(\%)$ \\
\hline Anacardium occidentale & Umbelliferea & Seeds & 36 \\
\hline Artemisia abrotanum & Compositeae & Aerial part & 34 \\
\hline Hypericum perforatum & GuttiferaeHypericaceae & Plant flowers & 34 \\
\hline Lavandula stoechasl & Lamiaceae & Aerial part & 34 \\
\hline Melissa officinalis & Lamiaceae & Leaves and shoot & 33 \\
\hline Salix alba & Salicaceae & Aerial part & 29 \\
\hline Heracleum persicum & Apiaceae & Fruits & 29 \\
\hline Teucrium polium & Lamiaceae & Aerial part & 28 \\
\hline Lepidium sativum L. & Cruciferae & Seeds & 26 \\
\hline Pimpinella anisum & Cruciferae & Seeds & 25 \\
\hline Peganum harmala & Zygophyllaceae & Aerial part & 24 \\
\hline Zingiber officinale & Zingiberaceae & Rhizomes & 24 \\
\hline Panax ginseng & Araliaceae & Roots & 23 \\
\hline Cydonia oblonga & Rosaceae & Seeds & 22 \\
\hline Valeriana hispida & Valerianaceae & Rhizomes & 22 \\
\hline Citrullus colocynthis & Cucurbitaceae & Fruits & 20 \\
\hline Ficus carica & Moraceae & Leaves & 18 \\
\hline Rosa canina & Rosaceae & Fruits & 16 \\
\hline Ocimum basilicum L. & Lamiaceae & Seeds & 15 \\
\hline Trigonella foenumgraecum & Leguminosae & Seeds & 15 \\
\hline Bunium persicum & Apiaceae & Seeds & 14 \\
\hline Papaver orientale & Papaveraceae & Aerial part & 14 \\
\hline Cichorium intybus & Compositae & Roots & 11 \\
\hline Althae officinallis & Malvaceae & Galls & 11 \\
\hline Hibiscus gossypifolius & Labiatae & Galls & 10 \\
\hline Ranunculus arvensisl & Ranunculaceae & Aerial part & 10 \\
\hline Portulaca oleracea & Portulacaceae & Seeds & 9 \\
\hline Artemisia dracunculus & Compositae & Leaves & 7 \\
\hline Citrus aurantium & Rutaceae & Galls & 6 \\
\hline Hibiscus gossypifolius & Malvaceae & Fruits and Calix & 5 \\
\hline Coriandrum sativum & Graminaceae & Stem soup & 4 \\
\hline Physalis alkekengi & Solanaceae & leaves & 4 \\
\hline Foeniculum vulgare & Umbelliferaeapiaceae & Fruits & 4 \\
\hline Carthamus oxyacantha & Compositae & Whole plant & 3 \\
\hline Ziziphus spinachristi & Rhamnaceae & leaves & 0 \\
\hline Alhagi camelorum & Fabaceae & Aerial part & 0 \\
\hline Fumaria parviflora & Fumariaceae & Aerial part & 0 \\
\hline Rubia tinctorium & Rubiaceae & Roots & 0 \\
\hline Terminalia chebulla & Combertaceae & Fruits & 0 \\
\hline Cordiamixa & Boraginaceae & Fruits & 0 \\
\hline Fraxinus excelsior & Oleaceae & Aerial part & 0 \\
\hline Vaccinium arctostaphylus & Ericaceae & Fruits & 0 \\
\hline Ocimum basilicum & Lamiaceae & Seeds & 0 \\
\hline Eucaliptus galbie & Eucalypteae & Leaves & 0 \\
\hline Anacardium occidentale & Apiaceae & leaves & 0 \\
\hline Malva sylvestris & Malvaceace & Galls & 0 \\
\hline
\end{tabular}

Table 2. Plants and their inhibitory effect on 4-Nitrophenyl $\beta$-D-glucuronide.

\begin{tabular}{l|l|l|l} 
Plants name & Family & Used part & Inhibition $(\%)$ \\
\hline Stevia & Asteraceae & leaves & 75 \\
\hline Cerasus avium & Rosaceae & Fruit Tails & 68 \\
\hline Rosa damascena & Rosaceae & Galls & 67 \\
\hline Calendula officinalis L. & Asteraceae & Whole plant & 67 \\
\hline Ziziphor atenuir & Lamiaceae & leaves & 58 \\
\hline Crataegus oxyacanta & Rosaceae & FruitsGalls and & 58 \\
\hline Origanum majorana & Lamiaceae & Whole plant & 49 \\
\hline Hyssopus officinalis & Lamiaceae & Plant flowers & 49 \\
\hline Scrophularia striata & Compositae & Aerial part & 45 \\
\hline Otostegia persica & Violaceae & Aerial part & 43 \\
\hline Zatari amultiflora & Lamiaceae & Aerial part & 35 \\
\hline Melissa officinalis & Lamiaceae & Leaves and Shoot & 34 \\
\hline Ipomoea tricolor & Convolvulaceae & Galls & 27 \\
\hline Tanacetum parthenium & Asteraceae & Galls & 27 \\
\hline Crataegus oxyacanta & Astraceae & Whole plant & 27 \\
\hline Papaver somniferum & Papaveraceae & Seeds & 25 \\
\hline Cucumis sativus & Cucurbitaceae & Seeds & 24
\end{tabular}


https://doi.org/10.33263/BRIAC111.79217931

\begin{tabular}{|c|c|c|c|}
\hline Plants name & Family & Used part & Inhibition (\%) \\
\hline Nigella sativa $L$. & Ranunculaceae & Seeds & 22 \\
\hline Lippia citriodora & Verbenaceae & leaves & 19 \\
\hline Sanguisorba minor & Geraniacea & Aerial part & 18 \\
\hline Glycyrrhiza glabra & Legumes & Aerial part & 16 \\
\hline Artemisia abrotanum & Compositeae & Aerial part & 16 \\
\hline Ranunculus arvensisl & Ranunculaceae & Aerial part & 16 \\
\hline Rhus coriaria & Anacardiaceae & Seeds & 14 \\
\hline Anacardium occidentale & Umbelliferea & Seeds & 13 \\
\hline Teucrium polium & Lamiaceae & Aerial part & 13 \\
\hline Avena sativa & Grasses & Seeds and shell & 11 \\
\hline Sizigium aromaticus & Caryophyllaceae & Aerial part & 10 \\
\hline Lavandula angustifolia & Lamiaceae & Whole plant & 10 \\
\hline Centaurea depressa & Grasses & Galls & 10 \\
\hline Citrus aurantium & Rutaceae & Galls & 10 \\
\hline Cotoneaster & Rosaceae & Gum & 10 \\
\hline Mentha piperita & Lamiaceae & leaves & 9 \\
\hline Phoenix dactylifera & Arecaceae & Galls and Floret & 9 \\
\hline Hypericum perforatum & Guttiferae Hypericaceae & Plant flowers & 9 \\
\hline Sesamum indicum & Pedaliaceae & Seeds & 8 \\
\hline Cichorium intybus & Compositae & Roots & 8 \\
\hline Olea europaea & Oleaceae & leaves & 6 \\
\hline Coriandrum sativum & Apiaceae & Aerial part & 6 \\
\hline Panax ginseng & Araliaceae & Roots & 5 \\
\hline Artemisia absinthium & Compositae & Leafy branches & 4 \\
\hline Rosa canina & Rosaceae & Fruits & 4 \\
\hline Ocimum basilicum L. & Lamiaceae & Seeds & 4 \\
\hline Rosa & Rosaceae & Galls & 3 \\
\hline Echium amoenum & Boraginaceae & Galls & 3 \\
\hline Astragalus adsccendens & Psyllidae & Gum & 3 \\
\hline Achillea wilhelmsii & Compositae & Aerial part & 3 \\
\hline Citrullus colocynthis & Cucurbitaceae & Fruits & 3 \\
\hline Trigonella foenumgraecum & Leguminosae & Seeds & 3 \\
\hline Artemisia dracunculus & Compositae & leaves & 3 \\
\hline Coriandrum sativum & Graminaceae & Stem soup & 3 \\
\hline Physalis alkekengi & Solanaceae & leaves & 3 \\
\hline Myrtus communis & Myrtaceae & leaves & 2 \\
\hline Cubeb berries & Piperaceae & Fruits & 2 \\
\hline Hypericum perforayum & Labiatae & Aerial part & 2 \\
\hline Urtica dioica & Urticaceae & Aerial part & 2 \\
\hline Rosmarinus officinalilis & Lamiaceae & Aerial part & 2 \\
\hline Mentha longifolia & Labiatae & Aerial part & 2 \\
\hline Heracleum persicum & Apiaceae & Fruits & 2 \\
\hline Peganum harmala & Zygophyllaceae & Aerial part & 2 \\
\hline Valeriana hispida & Valerianaceae & Rhizomes & 2 \\
\hline Bunium persicum & Apiaceae & Seeds & 2 \\
\hline Citrus aurantium & Rutaceae & Galls & 2 \\
\hline Hibiscus gossypifolius Mill. & Malvaceae & Fruits and calix & 2 \\
\hline Foeniculum vulgare & Umbelliferaeapiaceae & Fruits & 2 \\
\hline Carthamus oxyacantha & Compositae & Whole plant & 2 \\
\hline Equisetum arvense & Equisetaceae & Whole plant & 0 \\
\hline Arctium lappa & Asteraceae & Whole plant & 0 \\
\hline Medicago sativa $L$. & Legumes & Seeds & 0 \\
\hline Matricaria recutita & Composite & Galls & 0 \\
\hline Alhagi maurorum & Fabaceae & Gum & 0 \\
\hline Punica granatum & Punicaceae & skin & 0 \\
\hline Cinnamomum zeylanicum & Lauraceae & Roots & 0 \\
\hline Lactuca sativa $L$. & Compositae & Seeds & 0 \\
\hline Pimpinella anisum & Cruciferae & Seeds & 0 \\
\hline Zingiber officinale & Zingiberaceae & Rhizomes & 0 \\
\hline Cydonia oblonga Mill. & Rosaceae & Seeds & 0 \\
\hline Ficus carica & Moraceae & leaves & 0 \\
\hline Papaver orientale & Papaveraceae & Aerial part & 0 \\
\hline Althae officinallis & Malvaceae & Galls & 0 \\
\hline Hibiscus gossypifolius & Labiatae & Galls & 0 \\
\hline Portula caoleracea & Portulacaceae & Seeds & 0 \\
\hline Ziziphus spinachristi & Rhamnaceae & leaves & 0 \\
\hline
\end{tabular}




\begin{tabular}{l|l|l|l}
\hline Plants name & Family & Used part & Inhibition $(\%)$ \\
\hline Alhagi camelorum & Fabaceae & Aerial part & 0 \\
\hline Terminalia chebulla & Combertaceae & Fruits & 0 \\
\hline Cordia mixa & Boraginaceae & Fruits & 0 \\
\hline Fraxinus excelsior & Oleaceae & Aerial part & 0 \\
\hline Vaccinium arctostaphylus & Ericaceae & Fruits & 0 \\
\hline Ocimum basilicum & Lamiaceae & Seeds & 0 \\
\hline Eucaliptus galbie & Eucalypteae & leaves & 0 \\
\hline Anacardium occidentale & Apiaceae & leaves & 0 \\
\hline Malva sylvestris & Malvaceace & Galls & 0 \\
\hline Allium sativum L. & Lily & gland & 2 \\
\hline Polygonatum Orientale des. & Umbelliferae & Roots & 2 \\
\hline Camellia sinensis & Theaceae & leaves & 4 \\
\hline Lavandula stoechasl & Lamiaceae & Aerial part & 5 \\
\hline Salix alba & Salicaceae & Aerial part & 5 \\
\hline Lepidium sativum L. & Cruciferae & Seeds & 6 \\
\hline Fumaria parviflora & Fumariaceae & Aerial part & 8 \\
\hline Rubi atinctorium & Rubiaceae & Roots & 8
\end{tabular}

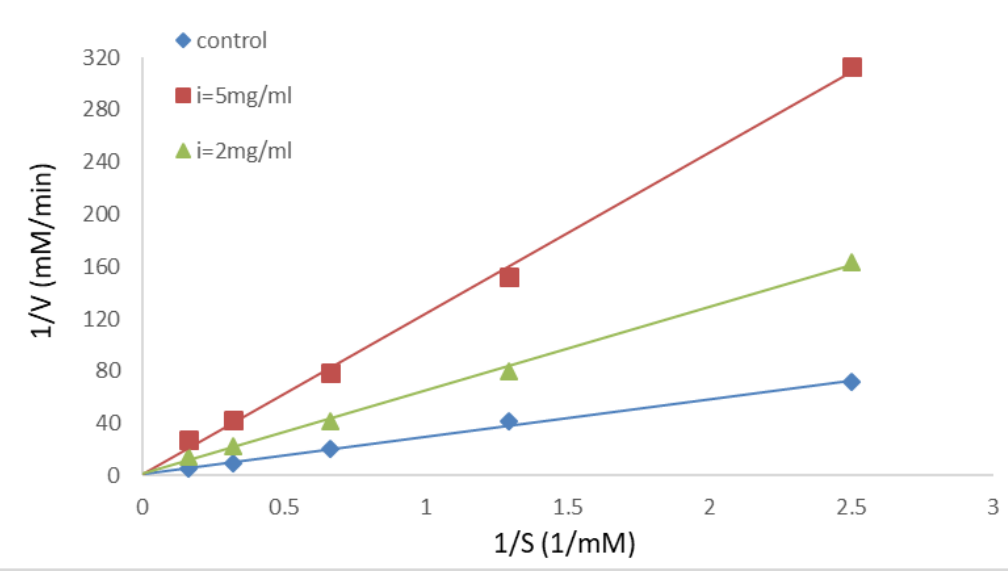

Figure 1. The Lineweaver-Burk plot of kinetic analysis for $\beta$-glucuronidase at two different concentrations of Rosa damascena (2.5 and $5 \mathrm{mg} \mathrm{mL}-1$ ) in the presence of 5 different Phenolphthalein glucuronide concentrations.

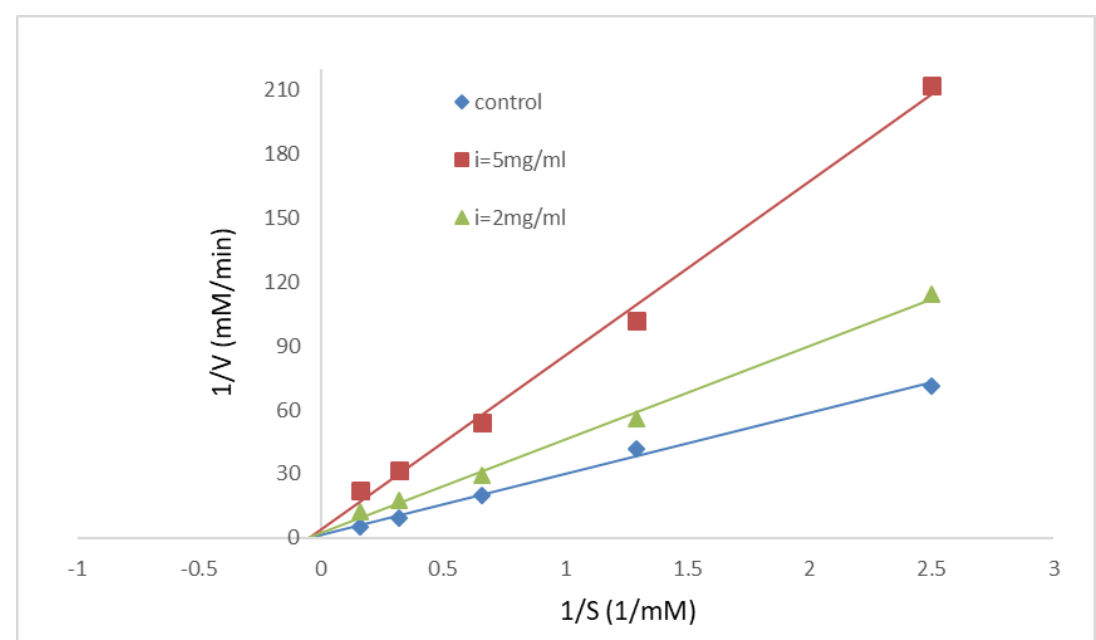

Figure 2. The Lineweaver-Burk plot of kinetic analysis for $\beta$-glucuronidase at two different concentrations of Ipomoea tricolor (2.5 and $5 \mathrm{mg} \mathrm{mL}-1$ ) in the presence of 5 different Phenolphthalein glucuronide concentrations. 


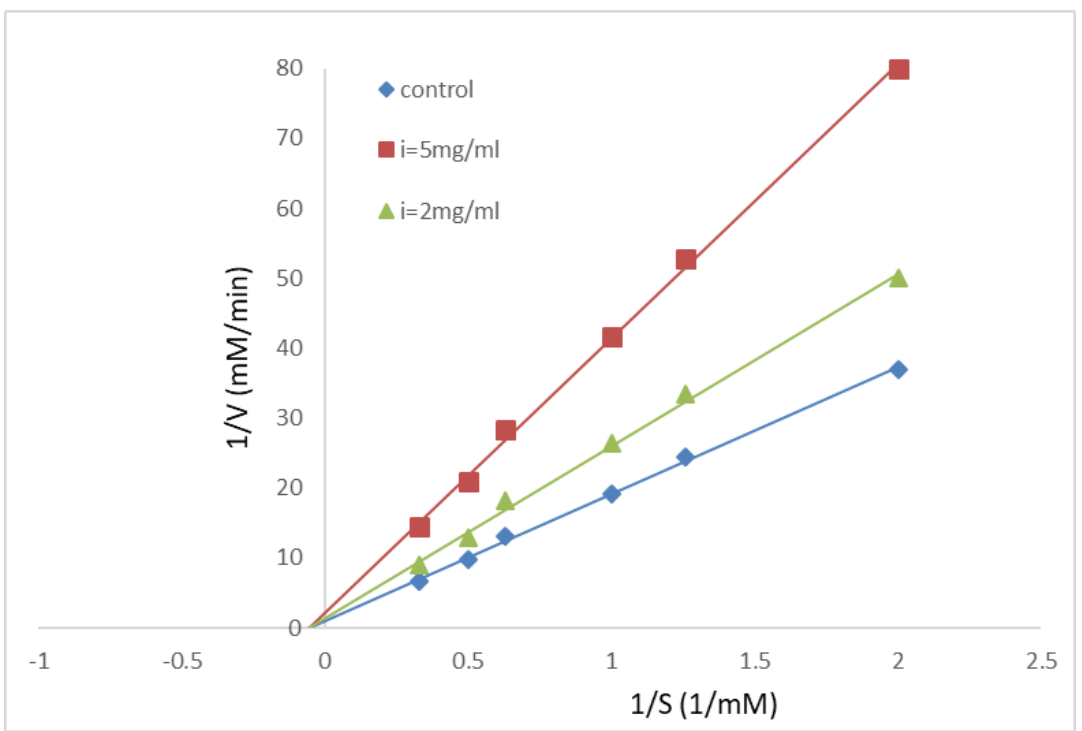

Figure 3. The Lineweaver-Burk plot of kinetic analysis for $\beta$-glucuronidase at two different concentrations of Stevia (2.5 and $5 \mathrm{mg} \mathrm{mL}-1)$ in the presence of 5 different 4-NPG concentrations.

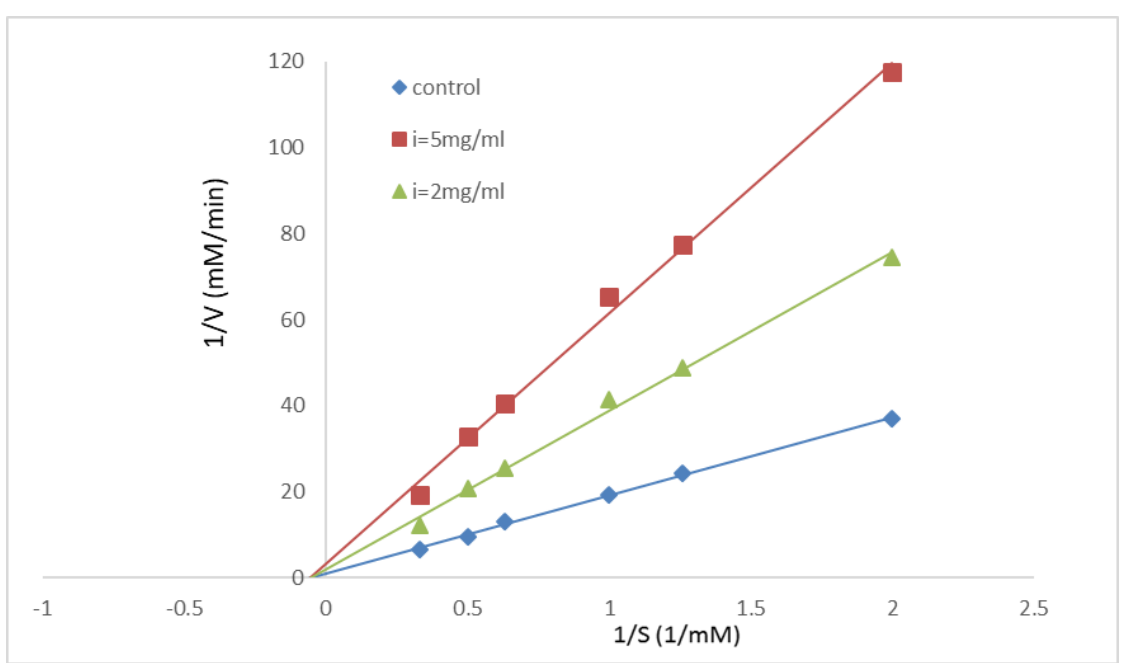

Figure 4. The Lineweaver-Burk plot of kinetic analysis for beta-glucuronidase at two different concentrations of Cerasus avium (2.5 and $5 \mathrm{mg} \mathrm{mL-1)} \mathrm{in} \mathrm{the} \mathrm{presence} \mathrm{of} 5$ different 4-NPG concentrations.

\section{Conclusions}

Hydro-methanolic extract of Rosa damascena, Ipomoea tricolor, Stevia, and Cerasus avium showed an inhibitory effect on $\beta$-glucuronidase. Since $\beta$-glucuronidase inhibition is considered as a biomarker of cancer pathology (especially colon cancer) and other disease condition including diabetes, therefore, its inhibitor must be evaluated in such disease for possible beneficial effects. The results provided by the current study scientifically validate the use of these plants in the treatment of the related diseases, and we need to evaluate the hydromethanolic extract of Rosa damascena, Ipomoea tricolor, Stevia and Cerasus avium for their beneficial effects in diseases mentioned above models.

\section{Funding}

This research received no external funding.

\section{Acknowledgments}

This study was financially supported by the Kerman Medical University Research Council. 


\section{Conflicts of Interest}

The authors declare no conflict of interest.

\section{References}

1. Seyedi, R.; Mirghafourvand, M.; Jannat Dost, A.; Mohammad-Alizadeh-Charandabi, S.; Asghari Jafarabadi, M. Relationship between Neonatal Skin Bilirubin Level and Severe Jaundice with Maternal, Childbirth, and Neonatal Characteristics. Iranian Journal of Neonatology 2019, 10, https://doi.org/10.22038/ijn.2019.33282.1478.

2. Fakhri, M.; Farhadi, R.; Mousavinasab, S.N.; Yosefi, S.S.; Hosseinimehr, S.J.; Azadbakht, M. Effect of Natural Products on Jaundice in Iranian Neonates. Jundishapur J Nat Pharm Prod 2019, 14, https://doi.org/10.5812/jjnpp.83042.

3. Roche. S.P.; Kobos, R. Jaundice in the adult patient. Am fam physician 2004, 69, 299-308.

4. Liu, W.; Liu, H.; Wang, T.; Tang, X. Therapeutic effects of probiotics on neonatal jaundice. Pak J Med Sci 2015, 31, 1172-1175, https://doi.org/10.12669/pjms.315.7921.

5. Gazzin, S.; Masutti, F.; Vitek, L.; Teribelli, C. The molecular basis of jaundice: An old symptom revisited. Liver Int 2017, 37, 1094-1102, https://doi.org/10.1111/liv.13351.

6. Zahed Pasha, Y.; Alizadeh-Tabari, S.; Zahed Pasha, E.; Zamani, M. Etiology and therapeutic management of neonatal jaundice in Iran: a systematic review and meta-analysis. World J Pediatr 2020, https://doi.org/10.1007/s12519-020-00339-3.

7. Bader, D.; Yanir, Y.; Kugelman, A.; Wilhelm-Kafil, M.; Riskin, A. Induction of early meconium evacuation: is it effective in reducing the level of neonatal hyperbilirubinemia? Am J Perinatol 2005, 22, 329333, https://doi.org/10.1055/s-2005-871529.

8. Awolade, P.; Cele, N.; Kerru, N.; Gummidi, L.; Oluwakemi, E.; Singh, P. Therapeutic significance of bglucuronidase activity and its inhibitors: A review. Eur J Med Chem 2020, 187, https://doi.org/10.1016/j.ejmech.2019.111921.

9. Whitehead, M.W.; Hainsworth, I.; Kingham, J.G. The causes of obvious jaundice in south west wales: perceptions versus reality. Gut 2001, 48, 409-413, https://doi.org/10.1136/gut.48.3.409.

10. Saluja, S.; Agarwal, A.; Kler, N.; Amin, S. Auditory neuropathy spectrum disorder in late preterm and term infants with severe jaundice. Int $J$ Pediatr Otorhinolaryngol 2010, 74, 1292-1297, https://doi.org/10.1016/j.ijporl.2010.08.007.

11. Maisels, M.J.; McDonagh, A.F. Phototherapy for neonatal jaundice. N Eng J Med 2008, 358, 920-928, https://doi.org/10.1056/NEJMct0708376.

12. Mahran, E.; Keusgen, M.; Morlock, G.E. New planar assay for streamlined detection and quantification of b-glucuronidase inhibitors applied to botanical extracts. Anal Chim Acta 2020, 4, https://doi.org/10.1016/j.acax.2020.100039.

13. Molan, A.L.; Saleh Mahdy, A. Iraqi medicinal plants: Total flavonoid contents, free-radical scavenging and bacterial beta-glucuronidase inhibition activities. IOSR-JDMS 2014, 13, 72-77, https://doi.org/10.9790/0853-13527277.

14. Wong, R.J.; Bhutani, V.K. Pathogenesis and etiology of unconjugated hyperbilirubinemia in the newborn. UpToDate. Waltham, MA: UpToDate, 2020.

15. Rothenberg, M.L. Irinotecan (CPT-11): recent developments and future directions-colorectal cancer and beyond. Oncologist 2001, 6, 66-80, Https://doi.org/10.1634/theoncologist.6-1-66.

16. Roberts, A.B.; Wallace, B.D.; Venkatesh, M.K.; Mani, S.; Redinbo, M.R. Molecular insights into microbial $\beta$-glucuronidase inhibition to abrogate CPT-11 toxicity. Mol Pharmacol 2013, 84, 208-217, https://doi.org/10.1124/mol.113.085852.

17. Hsieh, Y.T.; Chen, K.C.; Cheng, C.M.; Cheng, T.L.; Tao, M.H.; Roffler, S.R. Impediments to enhancement of CPT-11 anti-cancer activity by E. coli directed beta-glucuronidase therapy. PloS One 2015, 10, https://doi.org/10.1371/journal.pone.0118028.

18. Karak, S.; Nag, G.; De, B. Metabolic profile and $\beta$-glucuronidase inhibitory property of three species of Swertia. Rev bras farmacogn 2017, 27, 105-111, http://dx.doi.org/10.1016/j.bjp.2016.07.007.

19. Karak, S.; Das, S.; Biswas, M.; Choudhury, A.; Dutta, M.; Choudhury, K.; De, B. Phytochemical composition, $\beta$-glucuronidase inhibition, and antioxidant properties of two fractions of Piper betle leaf aqueous extract. J of Food Biochemistry 2019, https://doi.org/10.1111/jfbc.13048.

20. Perumal, A.; Naidu Krishna, S.B.; Sershen, K.; Pillay, K.; Govender, P. Phytochemical composition and biological investigation of Trichilia emetica Vahl. seed extracts. Letters in Applied Nano Bio Science 2020, 9, 1111-1116, https://doi.org/10.33263/LIANBS92.11111116.

21. Gandham, R.G.; Raji, P.; Rohan, B.; Divya Kumar, M.; Kripu Sharma, V.; Keerthana, D.; Karishma, S.; Iyappan, P.; Thrumurugan, R.; Samrot, A.V.; Ponnaiah, P.; Pattamadath, S.; Purayil, S.K.; Javad, P.T.M.; Prakash, P. Green synthesis and antibacterial activity of silver nanoparticles from the aqueous extracts of Cassia alata. Letters in Applied Nano Bio Science 2020, 9, 1037-1041, https://doi.org/10.33263/LIANBS92.10371041. 
22. Kumari, R.; Chandra Mishra, R.; Parkash Yadav, J. Antioxidant and cytotoxic studies of Acacia nilotica twig extract and their green synthesized silver nanoparticles. Letters in Applied Nano Bio Science 2020, 9, 975980.

23. Shim, S.B.; Kim, N.J.; Kim, D.H. $\beta$-Glucuronidase inhibitory activity and hepatoprotective effect of $18 \beta$ glycyrrhetinic acid from the rhizomes of Glycyrrhiza uralensis. Planta Med 2000, 66, 40-43, https://doi.org/10.1055/s-2000-11109.

24. Summart, R.; Chewonarin, T. Purple rice extract supplemented diet reduces DMH-induced aberrant crypt foci in the rat colon by inhibition of bacterial $\beta$-glucuronidase. Asian Pac J Cancer Prev 2014, 15, 749-755, https://doi.org/10.7314/APJCP.2014.15.2.749.

25. Nawaz, H.R.; Malik, A.; Khan, P.M.; Shujaat, S.; Rahman, A. A novel $\beta$-glucuronidase inhibiting triterpenoid from Paeonia emodi. Chem Pharm Bull (Tokyo) 2000, 48, 1771-1773, https://doi.org/10.1248/cpb.48.1771.

26. Joukar, S.; Askarzadeh, M.; Shahouzehi, B.; Najafipour, H.; Fathpour, H. Assessment of safety and therapeutic efficacy of rosa damascena L. and quercus infectoria on cardiovascular performance of normal and hyperlipidemic rabbits: Physiologically based approach. Journal of Toxicology 2013, 2013, https://doi.org/10.1155/2013/769143.

27. Acharya, J.; De, B. Bioactivity-guided fractionation to identify $\beta$-glucuronidase inhibitors in Nymphaea pubescens flower extract. Cogent Food \& Agriculture 2016, 2, https://doi.org/10.1080/23311932.2015.1134379.

28. Meister, A. Advances in enzymology and related areas of molecularbiology. Volume 70, John Wiley \& Sons, 2009.

29. Gholamhoseinian, A.; Sharifi-Far, F.; Rahimi-Naiini, M. Screening of methanol extracts of sixty plants from Kerman for their potential xanthine oxidase inhibitory activity. J Herbmed Pharmacol 2017, 6, 126129.

30. Kim, Y.M.; Jeong, Y.K.; Wang, M.H.; Lee, W.Y.; Rhee, H.I. Inhibitory effect of pine extract on $\alpha-$ glucosidase activity and postprandial hyperglycemia. Nutrition 2005, 21, 756-761, https://doi.org/10.1016/j.nut.2004.10.014. 